Papers and Proceedings of the Royal Society of Tasmania, Volume 112, 1978

(ms. received 8.12 .1977 )

\title{
A SIMPLIFIED GROWTH LINE REPLICATION TECHNIQUE
}

Scott Cane and Jim Stockton

ABSTRACT

An inexpensive and simple method of growth line replication is described and Mytilus planulatus, Ostrea angasi, Cellana solida and Subninella undulata are copied to provide a basis from which to assess the archaeological potential of these species.

\section{BACKGROUND}

Many species deposit mineralized tissues characteristic of and relating to predictable environmental rhythms. Examples of such deposits are found in the shell of molluscs and crayfish, in fish scales, bone, feathers, and wood. Mollusc shells are perhaps the most consistent depositors and as indexes for constructing local chronologies are of obvious archaeological value (Conover 1975).

Shell growth occurs as a result of secretion of inorganic (calcium carbonate) and organic (basically quinone-tanned protein) materials from the mantle of the shell fish. As the ultimate origin of these materials is the medium in which the she11 lives, any change in that environment will lead to different patterns of growth. These growth increments will be characteristic of the particular environmental situation (Wilbur 1972). For example, tidal patterns (Conover 1975, p.11) affect the absorption and deposition of materials for shell manufacture. As most shells calcify at high tide (submergence), tidal movements will define a characteristic pattern of shel1 growth. This environmental growth may be further complicated by seasonal variation in temperature (Pane1la and MacClintock 1968, p.71), spawning cycles (Richards 1946, p.37) and intermittent, catastrophic, events, for example storm activity causing dilution of sea water with fresh and temporary sedimentation. For these reasons she11 growth patterns are of significance to archaeologists. Using contemporary analogies and extensive research into the characteristics of growth 1 ines produced under certain environmental conditions, archaeologists can make conclusive statements regarding the seasonal movements of natives (Coutts 1970, p.147) and can potentially and literally describe the coastal environment in which these people lived.

There are several techniques used to render she11 growth patterns readable. Experimentation reported in this paper was done on the common edible musse1, Mytilus planulatus. Basically such techniques require the sectioning, polishing and replication of the she11 surface. Acetate (Stewart and Taylor 1965, pp.230-32) and plexiglass peels (Frank 1960, pp.498-500) are commonly used. Both techniques require critical polishing of the she11 surface and the maintenance of a perfect1y plane surface. This surface is essential as these relatively rigid materials must lie in direct contact with the shell section. This is a tiring and time consuning procedure and this paper describes a technique in which the level of the sectioned surface is of less consequence.

MOUNTING

Shells that have been burnt and weathered for a considerable time are relatively friable. This, allied with their porosity renders them difficult to handle unless impregnated with a highly fluid and hard setting resin. A most satisfactory method is to half fill a typical cylindrical mould with resin. This should be allowed to partially set so the shell can then be placed on this semi-hard layer and then completely covered with resin. Before mounting, each specimen should be cleaned by washing and drying in acetone. All moisture must be removed from the shell before mounting 
156

\section{Simplified Growth Line Replication}

as moisture tends to overheat and crack the cast. An acetone bath has the added advantage of removing many air bubbles from the shell. A high quality polyester casting resin is desirable as these are hard setting, transparent and have a low viscosity. Resins used in this experiment were trade named 'Star Cast' and 'Diamond Cast' (Arbee Handcrafts Centre Pty. Ltd., Melbourne) but we would expect any high quality commercially available casting resin and 'hardener' to be suitable.

The hardness of the cast is a direct result of the amount of catalyst used to set the resin. The greater the catalyst: resin ratio the harder the cast, and vice versa. The resin must be as hard (or soft) as the shell so that a continuous surface between the two is maintained when polishing. This degree of perfection requires experimentation and is difficult to attain. The resin:hardener ratio for this experiment was $8.7 \mathrm{~m} 1$ of hardener: $100 \mathrm{~m} 1$ of resin. This gave a curing time of $5 \frac{1}{2}$ hours at a temperature of $23^{\circ} \mathrm{C}$. This curing time may be decreased with an increase in temperature and a more suitable time of $1 \frac{1}{2}$ hours was found at $70^{\circ} \mathrm{C}$. An optimum level must be obtained for time to be used efficiently. Great care must be taken not to overheat and therefore, discolour and possibly crack the cast.

Mixing the resin and catalyst should be done very carefully to reduce the number of air bubbles trapped as these lessen the quality of the replica. Likewise the shell should be carefully placed in the resin so that air bubbles on the surface of the shell are not introduced. When the shell is in place, the mould and cast should be placed in a vacuum. The vacuum removes air bubbles from the cast and should be held or repeated until all air bubbles are removed. On releasing the vacuum, atmospheric pressure forces the resin into the shell (Davies and Hill 1968, p.235).

The vacuum is a very suitable method for removing air bubbles in slow setting casts. However, if the catalyst:resin ratio is too high the cast will rapidly overheat. This is because the removal of atmosphere prevents the absorption of heat given out by the exothermic setting reaction. Consequently the heat increases within the cast. This has two disadvantages. Firstly, overheating causes premature curing and the majority of air bubbles are trapped in the cast. Secondly the cast will most likely crack and if this does not occur the different cooling rates of the shell and resin will cause the cast to break from the shell surface. Replication is then impossible. (This variation j.n cooling rate is a problem with shells of varying thicknesses, for example, the umbo region of Mytizus and Notohaziotis).

\section{SECTIONING}

After the cast has cured, a section is cut at right angles to the growth increments (Panella and Macclintock 1968, p.67) from the umbo to the most remote point on the shell margin (Conover 1975, pp.30-1; Kioke 1973, p.24). The section may be cut with a diamond saw (Bissell 1957, p.417; Frank 1965, p.498) but this has no advantage over a hand held or band saw. The latter is in fact preferable due to the possible speed of sectioning. It is imperative that the cut be made as straight as possible. Any irregularities in the section are then removed with a flat faced file clamped horizontally in a vice.

\section{POLISHING}

When polishing the specimen it is necessary to place the abrasive material on a clean sheet of glass. This removes the risk of irregularities in the supporting surface damaging the shell surface. It is also desirable to change the polishing direction with each grade of paper so that one can see when the former scratches have been removed.

After sectioning and filing, the surface is hand polished with 600,800 and then 
1200 corundum paper. Each of these papers should be used until the scratches from the former are removed.

After the 1200 paper has been used a liquid or cream polish, e.g. Brasso or fine car polish, is used on a cloth in the same manner as the papers for at least 15-20 minutes. This time variation depends on the hardness of the cast. The quicker the resin sets the harder the cast and the greater the length of fine polishing. This treatment reduces the majority of coarse scratches but does not remove fine microscopic pits. These may be removed by finer grades of polish. Six micron diamond dust was used in this experiment; however, this procedure is time consuming and with diamond dust at $\$ 32$ per 5 grams may often be impractica1. Etching to remove these pits is preferable as it is cheaper and much faster.

\section{ETCHING}

At this stage the shell section is generally etched with HCl. This serves two purposes, firstly decalcifying the shell and thus raising the organic framework of the shell to be copied (Conover 1975, p.24) and secondly the etch serves to "polish" the she11 surface. The process of replication described here incorporates the etching material within the stain, thus excluding this stage in the procedure.

\section{STAINING}

The only calcium stain found suitable for glue replication was Harris haematoxylin (for preparation see Friedman 1959, p.93). This stain is relatively slow and a period of 15 minutes is required to stain $M$. plarulatus. The acetic acid content of this stain serves as an etch obviating the $\mathrm{HCl}$ etch used in acetate and plexiglass replications.

\section{REPLICATION}

The excess stain should be washed off and the surface dried with the aid of alcohol and a gentle air current. Care must be taken not to smudge the stained and etched surface. The glue used for replication was called "Mister McGloo" and was applied as evenly as possible, with the thickness being the minimum possible, as the thickness of glue decreases with setting. The only disadvantage of this method is the four hour setting time. This is however less of a disadvantage than the problems created by the polishing requirements for the other methods. When lifting the peel from the section, the peel should be removed slowly and carefully in order to preserve an accurate record of the growth structures and to ensure that parts of the glue do not stay in the shell framework. The final result is a very clear replication of the shell surface. This should be mounted on a glass slide with the copied surface up. Sticking tape is sufficient for this purpose.

\section{APPLICATION}

Having refined a suitable replication technique it was a relatively simple process to investigate the potentials of different shell species for growth line analysis. The common mussel Mytizus planulatus; mud oyster Ostrea angasi; orange rimmed limpet Celzana solida and wavy turbo Subrinelza undulata were investigated.

Because of the brevity of the investigation any conclusions must be considered as tentative.

\section{Mytizus planulatus}

Growth patterns in this species are very clear and the regularity and repetition of these patterns suggest a shell of considerable value to archaeologists. 
Cellana solida

The replication process for the orange-rimmed limpet is identical to that for Mytizus planulatus. Having studied only two individuals of this species it is difficult to make any definite archaeological suggestions. Apparently the outer (prismatic) layer of $C$. solida is eaten by a white parasitic fungus and consequently provides a very incomplete record.

The nacreous layer consists of definite bands which run obliquely to the base of the shel1. We suggest these are a result of variations in growth and as only alternate 'increments' absorbed the stain we suggest these are calcium rich. The overall pattern may therefore present periods of stress and non-stress. However, these bands are not continuous and are best described as lenses of calcium rich incremental growth. Their archaeological potential is suspect and only continued research will accurately assess their potential.

Ostrea angasi

Ostrea angasi (mud oyster) is abundant in present day estuaries and estuarine middens. However, it appears unsuitable for growth ring study.

The shell is difficult to mount because the finely laminated structure holds large qualities of moisture and numerous small air spaces. In the latter case this is most important if the plexiglass or acetate methods of replication are to be used, as numerous air bubbles (which result in holes in the cast) occur at the shell edge. Premature evaporation of the solvent then occurs and the replication is very poor. In the former case the moisture often causes the resin cast to crack and this invariably occurs where a section is to be taken. The interior of these shells also contain large air spaces and these render any method of replication hopeless.

There also appear to be lenses of rich calcium material in 0 . angasi. These are very soft and make polishing difficult and, more importantly, parts of the shell are removed by the replicating material so high power magnification of the peel surface is prevented.

The crystalline structure of the mud oyster also seems to be unsuitable for growth ring study as it consists of what appear to be crystalline lamellae. This concentration of fine layers may present a shell in which the growth patterns are difficult to interpret.

Subninezla undulata

The shell of the wavy turbo presents obvious embeding difficulties. Therefore experimentation was done with the operculum of the species and this proved successful. Mounting is a very simple process and the replication procedures are identical to M. planuzatus, except the operculum requires a $1 / 2$ hour exposure to Harris haematoxylin.

The interior of the operculum consists of three different structures, the outer region of which appears to have very clear growth lines. The ease of mounting the operculum and the presence of this species in a majority of coastal middens must present a species of significant archaeological value.

\section{CONCLUSTON}

The use of the 'glue peel' has two primary advantages. Initially the materials required for shell replication are easily obtained and inexpensive. Secondly, this method excludes the major practical drawback - the difficulty involved in maintaining both a level and highly polished section. As the peel substance is liquid, any curves or undulations (which are exceedingly difficult to avoid) in the polished face pose no problem as this viscous liquid adequately covers all required surfaces. 
Scott Cane and Jim Stockton

The financial and practical savings of this method renders shell replication possible for any technician whether they be in well equipped laboratories or in a school class-room. The relative simplicity of replication and excellent resolution obtained with this technique render it most suitable for growth ring study.

The procedure outlined should facilitate investigation into the growth patterns produced by different environmental factors.

\section{REFERENCES}

Bisse1, H.J., 1957: Combined Preferential Staining and Cellulose Pee1 Techniques. J. Sedim. Petrol., 27 (4), 417-420.

Conover, K.J., 1975: Malachronology in the service of archaeology. A.N.U. Res. School of Pacific Studies. Unpub. Pap.

Coutts, D.J.F., 1970: Bivalve growth patterning as a method of seasonal dating in archaeology. Nature, 226, No. 5248, 487.

Davies, J.T. and Hil1, R., 1968: Stained dry cellulose pee1s of ancient and recent impregnated carbonate sediments. J. Sedim. Petroz., 38, 234-237.

Frank, R.M., 1960: An improved carbonate peel technique for high powered studies. J. Sedim. Petrot., 35, 498-500.

Friedman, G.M., 1959: Identifications of carbonate minerals by staining methods. J. Sedim. Petrot., 29 (1), 87-97,

Kioke, H., 1973: Daily growth lines of the clam Meretrix Zusoria. J. Anthrop. Soc. Nippon, 81 (2), 123-138.

Panella, G. and MacClintock, C., 1968: Biological and environmental rhythms reflected in molluscan shell growth. J. Paleobot., 24, 64-81.

Richards, O.W., 1946: Comparative growth of Mytizus califomionus at La Jo1la, California and Mytilus edulis at Woods Hole. Ecology, 27, 370-372.

Stewart, W.N. and Taylor, T.N., 1965: The Peel Technique in Kummel, B. and Raup, D., (Eds): HANDBOOK OF PALEONTOLOGICAL TECHNIQUES, Freeman, San Francisco. 224-232.

Wilbur, K.M., 1972: Shell formation in mollusca in Florkin, M. and Scheer, B.T., (Eds): CHEMICAL ZOOLOGY, 7, 103-145. Academic Press, New York. 